\title{
An Analysis on Application Prospects and Problems of IT in Hospital
}

\author{
Hu Shengli \\ Information center of Central Hospital of Cangzhou, Cangzhou, 061001,Hebei Province, China \\ hushenglihsl@139.com
}

Keywords: RFID technology; IT; hospital

\begin{abstract}
The article first discusses the Internet has broad application prospects in the field of medicine and health care. Secondly, it points out that the application faces several problems at present, such as, loss of medical standards, poor IT technology, security threat, lack of site code resources, lack of mature business model, industrialization and talents and so on. In conclusion, the article puts forward the solutions to the above-mentioned problems and then analyzes on them. The standard of IT technology in medical field should be set up. We should make construction of industry chain and laws and regulations, set up research institutions, cultivate compound talents, solve the cost problem and so on, which will have a great significance in promoting the wide use of IT technology in medical informatization and construction of a high-quality digital hospital.
\end{abstract}

\section{Introduction}

As arranged, Internet connects itself with any item through such sensing equipments as the radio frequency identification (RFID) device, infrared sensors, global positioning system and laser scanner. Then they exchange information and communicate with each other, thus realizing intelligent identification, location, tracking, monitoring and management.

Internet started earlier in Europe, the United States, Japan and South Korea, who pay close attention to the application of IT in medical and health field. Under this circumstance, our government issued A Development Plan for the Application of IC Card of 11th Five-Year Plan in Health System in 2008 in order to promote construction of One-Card in health field. Opinions on Deepening Medical and Health System Reform was introduced in 2009, which put forward to increase the application of RFID technology [1]. In the same year, the Ministry of Health held a conference discussing application of RFID in the field of medical and heath care with a view to promoting the Internet application in medical and health field. The Prime Minister, Wen Jiabao, mentioned in the third meeting of the 11th National People's Congress On March 5, 2010 that research and application of Internet should be speed up and more investment and policy support should be put on emerging industries with strategic importance.

In view of the above, our government attaches great importance to the Internet technology in medical field. The Internet, as a strategic emerging industry has a very broad application prospect in the field of medical information. We will take several typical applications as example in the following chapters.

\section{Application Prospects of IT in Hospital}

A Whole-process Monitor and Anti-counterfeiting of Drugs and Consumables. Application of Internet technology can strengthen the management of medicines and medical consumables. RFID can trace on raw materials, producing area, finished product batches, price, distribution, usage and so on. In the meantime, label information is stored in the database. We can compare the tag data with the information in the database when there are problems. As a result, fake and inferior products can be effectively prevented to ensure the safety of the quality of the drugs and consumables from the source.

Management of Medical Waste. RFID technology can help to trace on the whole process of medical waste from the hospital to the concerned company, which not only makes the responsibility 
of two units clear, but also ensure the complete processing of medical waste and avoid potential hazardous medical recycling [1].

Management of Medical Equipment. Medical equipment with RFID tag can trace on and manage the surgical instruments, smart sponge, implant materials and other instruments which are highly priced, radioactive and sharp[2].

Easy Management of Special Patients. RFID technology can easily identify the patients who are emergent, difficult to communicate and unconscious and save the precious time of treatment for them. As for patients with infectious disease, we can monitor them such as body temperature, pulse and other vital signs by RFID, thus resulting in not only providing the personalized treatment but also avoid the risk of infection and spread. The positions of mental patients, the elderly and children can be accurate so as to prevent them from being lost.

Effective Management of Blood. The whole process of blood can be traced on if we label RFID on qualified bags, which can not only avoid the barcode of small capacity but also ensure the safety of blood [1].

Convenient for Medical Staff and Patients. RFID is convenient both for medical staff and patients. The medical staff can quickly query all previous medical records of patients, including medical history, history of allergies, drugs used and the inspection list, so that a lot of repetitive work can be reduced and the medication is ensured. On the other hand, patients can log on the Internet through the mobile phone or other information terminal to register, consult, interrogate, query their health and etc.

Carrying out Remote Medical Treatment. Medical staff can realize remote diagnosis through the Internet. Remote sensing technology can collect patient's various life characteristics such as body temperature, blood pressure, heart rate, ECG, respiration and pulse. And also it can make 24-hour monitor and provide medical treatment for patients such as health care and dietary. So RFID can effectively monitor chronic diseases and locate on the patients, which will save the time when it is emergent.

Mobile Care. Information such as name, age, gender and history of drug allergy can be stored in the patients' wristbands. Nurses can get the above-mentioned information by PDA and then identity their patients, execute the doctor's advice through the wireless network and record patients' information.

Preventing the Babies from Being Stolen. Babies can be positioned and traced on by electronic tags on their wrists, which are harmless and can launch the RFID signal. The door will be open when the identification of the baby is right. All the access time and identity information are recorded in the database and an alarm is given when someone attempts to steal a baby.

Application in Response to Disaster System. Data of medical equipments in operating room, ICU and laboratory can be collected and analyzed by electronic tags. The potential accidents will be given an alarm. In addition, we can schedule the unified resource of the disaster scene.

\section{Application Problems of IT in Hospital}

Non-establishment of Medical Standard. There is no global standard of RFID. It is difficult to enter into comprehensive stage of industrialization due to lack of the industry standard. All walks of life should be developed in a unified technology platform. In addition, the standard is essential to Internet in medical industry. the global unified technical standards, also must be solved for the unity of the medical and health industry standards. Standard is not unified will severely restricts the industrial layout and the promotion.

Poor IT Technology. Our technology in Internet is poor compared with Europe and the United States and other countries. They started early, mastered the core technology and made a lead in depth, breadth and intelligence of application. The cost is high because the core technologies are monopolized by foreign companies when we embed electronic labels in medical apparatus and instruments and consumables which will make trouble in promoting IT in medical and healthy field [4]. 
Lack of Security. Application of Internet in the field of medicine and health will inevitably pose a threat to patients' privacy information and medical data for RFID tag is designed in an open way, cloud services are transparent and the traditional network security mechanism cannot be applied to the wireless sensor network. In order to ensure the security of Internet, a new security agreement is needed [5].

Lack of Code Addresses. Applications of IT is based itself on the code addresses which is in a large quantity and plentiful. However, only $6 \%$ of the IP addresses are in use at present. With the development of the Internet technology, our country will face the danger of running out of IP addresses. While it is possible to realize the transition from IPV4 to IPV6, but IPV6 application in our country has been lagged behind. Limited code addresses will seriously hinder the development of Internet in our country unless measures are taken [6-11].

Immature Business Model. At present the Internet technology has been applied in the identification, environment monitoring, logistics tracking, and other fields. The Internet technology has been preliminarily applied in some hospitals, but on the whole, has yet been into a mature business model.

Industrialization. It industry pattern is still in grope, Under the guidance of our government, manufacturers of RFID electronic tag and sensor and mobile operators should be gathered tighter. It will be a long time for us to achieve industrialization. Thus, the Internet is difficult to be widely used in the medical field without industrialization.

Lack of IT Talents. A lot of IT talents are needed if IT is widely used in medical industry, which should not only grasp the Internet but also be familiar with the process of hospital [4].

\section{Solutions to IT Applications in Hospital}

Making out IT Standards in Medicine. China, together with the United States, Germany, Britain, Korea and other countries, is dominant in making out international IT standards. Therefore, we should invest on it to make research and development, conquer the highest position in technological innovation and then effect a large number of practical applications. In the meanwhile, we should research, develop and promote relevant international standards. And national relevant departments should endeavor to make coding standards and regulations in the health field according to the characteristics of the medical industry to speed up the application of Internet in the medical industry.

Construction of Industry Chain. In promoting the Internet application in hospitals, our government should play a leading role to carry out IPV6's national action plan as soon as possible, thus meeting the requirements of China's rapid development in the future. We should seize market opportunities by investing on research of intelligent processing software and sensor chip and getting independent intellectual property rights of them.

Set-up of IT Research Institutes. Professional IT research institutes aim at solving problems in the process of application such as security threats, the clock out of sync, limited protocol algorithm and so on [5]. We should get independent intellectual property by mastering the core technology such as RFID, sensor, intelligent processing software, network and communication and nano materials, thus making achievements with independent intellectual property and developing high-end sensor. In the meantime, a study on IT security should be effected in order to solve the problems arising from the application of Internet such as virus, hacker attacks, privacy and etc.

Construction of Laws and Regulations. Laws and regulations regarding IT in remote medical treatment, medicine and equipment management and the privacy protection are required in order to make assurance of the health development of the Internet in hospital. In all, the laws and regulations must step up with the new technology.

Cultivation of IT Talents. Internet is a complex system engineering which needs the people who grasp not only communication, network and computer but also the process of hospital. Wuxi sensor network engineering center was established in 2009, whose job is to research the Internet technology and cultivate the concerned talents. 31 colleges and universities in our country have set up majors related to Internet till December 4, 2011. the universities, research institutes and enterprises should 
play a leading role in the cultivation of professional talents. In addition, training institutions are needed. Corporate practice is an important part of the cultivation of talents, thus, the talents will meet the requirements of the enterprises.

The Cost. Internet technology is poor in hospital and the cost of application in the field of health is high. In view of the above, our government should make a major investment. It is not appropriate for the hospital to make a large investment for it will increase the cost of medical treatment.

\section{Summary}

Internet is still in its infancy in our country. Wide use of IT in medical and health field arise many problems. But with the development of science and the progress of technology, Internet will play an important role in improving the sharing of medical resources and reducing the medical cost. In the future, the Internet will bring about the innovation of concept more than the progress of technology in the field of medicine and health care.

\section{References}

[1] Zhu Min, Chen Xiaoli, Zheng Lingxiang,Etc, Design of POCT quality management system based on the Internet of things [J].Chinese Journal of Clinical Laboratory Science,35(2017) 405-408.

[2] Gao Fan, Yu Jiaping. Investigation on military hospitals deepening service for army based on modern information technology innovation [J]. Practical Journal of Medicine \& Pharmacy,34(2017) 374-375,378.

[3] Zhang Jing, Shui Liming, Jiang Zhiqin,Etc. SWOT analysis and countermeasure research of Ningbo cloud hospital [J]. Chinese Journal of General Practice,15(2017) 338-342.

[4] Liu Yuquan, Qian Qiang. APPLICATION OF INTERNET OF THINGS TECHNOLOGY IN HOSPITALS[J]. Chinese Hospital Architecture \& Equipment,5(2017) 97-99.

[5] Yao Wenpo, Wu Min, Shen Huaqiang,Etc. Application of Internet of Things technology in hospital management[J]. Chinese Medical Equipment Journal,38(2017) 136-139.

[6] Zhang Xueying, Wang Junxin, Dong Wenyang. Design and practice of mobile medical system based on IOT technology [J]. China Health Care \& Nutrition,27(2017) 314.

[7] Huang Jie, Pan Yujia. Design of the Newborn Safety Management System by Combining Internet of Things and Wireless Local Area Network [J]. Journal of Medical Intelligence,38(2017) 33-35.

[8] Yao Wenpo, Wu Min, Shen Huaqiang,Etc. Design and Analysis of Hospital Warehouse Management System Based on RFID [J]. China Medical Devices,32(2017) 137-140.

[9] Chen Huangyu, Wang Juan. Design of Mobile Monitoring System based on Internet of Things [J]. China Medical Devices,32(2017) 116-119.

[10]Liu Meiling. Research on Emergency Model of Regional Coordination Cardiovascular Disease Based on Mobile Internet of Things [J]. China Health Industry,14(2017) 14-15.

[11]Yao Hongwu, Liu Bowei, Suo Jihong,Etc. Progress of application of internet of things technology in management of nosocomial infections [J]. Chinese Journal of Nosocomiology,27(2017) 3131-3134. 